\title{
Hantavirus Pulmonary Syndrome in the United States
}

\author{
Marilyn Fabbri, MD, and Melanie J. Maslow, MD
}

\author{
Address \\ Infectious Diseases Section (III), Veterans Affairs New York Harbor \\ Health Care System, 423 East 23rd Street, New York, NY 10010, USA. \\ E-mail: melanie.maslow@med.va.gov \\ Current Infectious Disease Reports 200I, 3:258-265 \\ Current Science Inc. ISSN I523-3847 \\ Copyright $(\subset 2001$ by Current Science Inc.
}

Since the first outbreak of hantavirus pulmonary syndrome (HPS) in 1993, understanding of the vast distribution and potential impact of hantaviruses has grown. At least 277 cases of HPS have been documented in the United States. The full clinical spectrum has yet to be elucidated, and one outbreak suggested the possibility of person-to-person transmission. New research has identified the $\beta-3$ integrins as cellular receptors for hantaviruses and has determined the pivotal role of the immune system in pathogenesis. Rapid diagnosis has been facilitated by a new immunoblot assay to detect Sin Nombre virus infection. Treatment remains primarily supportive; however, a placebocontrolled trial of ribavirin is ongoing. Extracorporeal membrane oxygenation may be a potential therapy in severe cases; inhaled nitric oxide needs further study. Vaccines developed against hantaviruses associated with hemorrhagic fever and renal syndrome might be effective against HPS-associated strains.

\section{Introduction}

An outbreak of an acute febrile illness characterized by respiratory failure, hypotension, and shock was reported by physicians in the Four Corners region of the United States in May 1993. A previously unknown hantavirus was subsequently identified as the cause [1]. Unlike the previously characterized hantaviruses, which cause fever, hemorrhagic manifestations, and acute renal failure (hemorrhagic fever with renal syndrome), the newly named hantavirus pulmonary syndrome (HPS) presented with a brief prodromal illness followed by rapidly progressive noncardiogenic pulmonary edema [2]. The earliest known case of HPS to be confirmed by serology occurred in Utah in 1959 [3].

The mortality rate associated with HPS is $40 \%$ to $60 \%$, although mild and even asymptomatic cases are now recognized. In the United States, HPS is caused by at least four hantaviruses: the Sin Nombre virus (SNV, responsible for the Four Corners outbreak), the Black Creek Canal virus (isolated from the Florida cotton rat) [4], the Bayou virus in Louisiana [5], and the New York 1 hantavirus [6]. Several other closely related viruses have been identified in Canada and South America. An estimated 1000 to 2000 cases of hantaviral infections occur each year worldwide.

\section{Case Definition}

Hantavirus pulmonary syndrome is a febrile illness characterized by bilateral diffuse interstitial edema on chest radiography in a previously healthy person; respiratory compromise develops within 72 hours of hospitalization. The syndrome should be suspected in cases of unexplained fatal respiratory illness in which autopsy examination shows noncardiogenic pulmonary edema without an identifiable cause. Clinical suspicion is confirmed with serology, detection of hantaviralspecific RNA by polymerase chain reaction, or detection of hantaviral antigen by immunohistochemical testing of clinical specimens (Table 1).

\section{Virology}

Hantaviruses are lipid-enveloped, tri-segmented, negativesense RNA viruses belonging to the Bunyavirus family. The genus Hantavirus was named for the first virus isolated in 1976 from a striped field mouse near the Hantaan River in South Korea. Unlike other members of the Bunyavirus family that are transmitted to humans by arthropod vectors, hantaviruses are transmitted via inhalation of viruscontaminated aerosols of rodent saliva, urine, and feces.

The hantavirus genome consists of three segments. The large (L) segment encodes a protein with replicase, transcriptase, and endonuclease activity. The medium (M) segment encodes a precursor protein that is processed to form the surface glycoproteins G1 and G2, one or both of which play a role in viral neutralization, fusion of infected cells, and hemagglutination. The small (S) segment encodes the nucleocapsid protein, which forms the filamentous helical nucleocapsid of this virus and elicits the humoral immune response.

Hantaviruses have been characterized by genetic and antigenic methods. Point mutations appear to account for most of the genetic drift among hantaviruses [7]. RNA viruses with segmented genomes, such as influenza virus, 


\title{
Table I. Definition of hantavirus pulmonary syndrome
}

\author{
Case definition \\ Febrile illness occurring in a previously healthy person, characterized by unexplained acute respiratory distress syndrome or \\ bilateral interstitial infiltrates developing within I week of hospitalization; respiratory compromise requiring supplemental \\ oxygen, or \\ Noncardiogenic pulmonary edema at autopsy with no identifiable specific cause of death \\ Case confirmed \\ Patient with a compatible clinical illness and one of the following: \\ Hantavirus-specific lgM or fourfold increase in IgG titers, or \\ Positive results on polymerase chain reaction for hantavirus ribonucleic acid, or \\ Positive results on immunohistochemistry for hantavirus antigen
}

Adapted from MMWR [49].

can reassort their RNA segments when dual infection of a target cell occurs. Genetic reassortment has been demonstrated in nature and in vitro for Bunyaviridae that cause arthropod-borne disease, but little information is available about HPS-associated strains.

Two previous studies analyzing genomic SNV RNA sequences from humans and rodents suggest that the RNA segment has been reassorted in nature between SNV genetic variants $[8,9]$. Rodriquez et al. [10] analyzed progeny virus from co-cultures of closely related SNV strains with identical rodent hosts and of SNV and Black Creek Canal virus, which have different rodent hosts. Reassortants were observed frequently between closely related strains but were rare between hantaviruses with different rodent hosts. These data suggest that genetic reassortment in nature may contribute to the emergence of new hantaviruses.

Hantaviruses are subdivided into genogroups on the basis of nucleotide sequence analysis of $\mathrm{S}$ and $\mathrm{M}$ segments. Each genogroup usually infects a single rodent species. The New World hantaviruses are more closely interrelated than the Old World viruses, and vice versa. The HPS-associated hantaviruses belong to the New World group; these include Sin Nombre, Bayou, Black Creek Canal, New York, and Andes viruses (Table 2). The Old World group includes Hantaan, Dobrava, Seoul, and Puumala viruses, which cause hemorrhagic fever with renal syndrome (HFRS).

\section{Ecology and Transmission}

Each hantavirus is maintained in nature by asymptomatic infection of a single rodent species. Hantaviral infection has been documented in cats, birds, and bats, but these hosts play no documented role in human transmission [11•]. Rodents shed infectious virus in saliva, urine, and feces for prolonged periods despite the presence of neutralizing antibody $[12 \bullet \bullet]$. Rodents are infected horizontally through biting and scratching other rodents, as reflected in a higher prevalence of infection in older male mice [13].

Phylogenetic relationships between virus and rodent host demonstrate a co-evolution over thousands of years. Studies in which hantaviral gene sequences are compared to rodent mitochondrial gene sequences allow nearly identical phylogenetic trees to be constructed [14]. Several hantaviruses can be found in one geographic area, each circulating within its specific rodent host.

Human transmission usually occurs after inhalation of aerosols of infected rodent excreta. The virus may also be transmitted after rodent bites, after inoculation of infected rodent excreta through skin lesions or eyes, and possibly through ingestion of contaminated food or water [15]. The incubation period ranges from 4 to 42 days (mean range, 12 to 16 days).

The risk for human disease is proportional to the frequency of exposure to infected rodents. Rodent populations may vary seasonally and can increase tenfold in 2 months. Navajo oral tradition recognized outbreaks of a disease similar to HPS when large rodent populations were present [16]. In the fall of 1992, heavy precipitation in a previously drought-stricken area expanded the deer mouse population tenfold by the time of the May 1993 outbreak [17].

Vertical transmission of hantaviruses in pregnant women with HPS has not been demonstrated. In a recent review of five cases of HPS in pregnancy [18•], there was no pathologic or immunohistochemical evidence of HPS in placental and fetal tissue from two in utero deaths, or serologic evidence of hantaviral infection in the two live births. This review identified one potential case of perinatal transmission of HFRS from Korea. No evidence suggests transmission via breast feeding in humans or animal models.

The issue of person-to-person transmission is controversial. In a study of 266 health care workers exposed to patients, to patients' body fluids, or to laboratory specimens during the 1993 outbreak, serologic testing done within 6 weeks of exposure found no evidence of infection [19]. Serosurveys performed on household contacts of patients with HPS revealed no IgM antibody reactive with SNV [20].

Person-to-person transmission may have occurred during an outbreak of HPS caused by the Andes virus in El Bolson, Argentina, in 1996. Polymerase chain reaction products of partial sequences of M and S viral RNA segments were studied from clinical samples to assess homology. Samples from 16 epidemiologically linked cases from this outbreak were 


Table 2. Hantaviruses associated with hantavirus pulmonary syndrome
\begin{tabular}{|lll|}
\hline Virus & Host & Distribution \\
\hline Andes & Oligoryzomys longicaudatus & Argentina, Chile \\
Bayou & Oryzomys palustris & Southeastern United States \\
Black Creek Canal & Sigmodon hispidus & Southeastern United States \\
Hu39694 & Unknown & Argentina \\
Juquitiba & Unknown & Brazil \\
Lechiguanas & Oligoryzomys flavescens & Argentina \\
Laguna Negra & Calomys laucha & Paraguay, Bolivia \\
Monongahela & Peromyscus maniculatus & United States, Canada \\
New York & Peromyscus maniculatus & Eastern United States \\
Oran & Oligoryzomys longicaudatus & Argentina \\
Sin Nombre & Peromyscus maniculatus & United States, Canada \\
\hline
\end{tabular}

analyzed, including samples from two physicians (from areas $130 \mathrm{~km}$ and $1400 \mathrm{~km}$ away from El Bolson) who had been in contact with the El Bolson cases and developed HPS during the same period. Nine other nonepidemiologically linked samples were analyzed; among these were samples from contemporary cases from the same area and cases from other areas and times. The 16 epidemiologically linked cases contained the same partial sequences in the $S$ and $M$ segments analyzed, whereas the sequences from the nine other cases differed. This finding suggested that person-to-person transmission of Andes virus had occurred. Of note, contact with rodents was not evident during this outbreak. No rodents were captured inside the households of patients in El Bolson, and rodent-trapping attempts around the town were unsuccessful $[21 \bullet]$.

In the United States, only universal precautions are recommended for patients suspected of having HPS. In areas where Yersinia pestis is endemic, patients are often placed in respiratory isolation until pneumonic plague is ruled out because symptoms may mimic those of HPS.

\section{Epidemiology}

From May, 1993 through December 7, 2000, 277 cases of HPS were reported in the United States [22]. Cases have been reported in 31 states (Fig. 1) and in Canada and South America. The overall US mortality rate is $38 \%$, compared with $52 \%$ in the first 100 US cases [3] (Table 3). The mean patient age is 38 years. Men make up 59\% of the total cases. Caucasians account for $77 \%$ of cases, and Native Americans for $20 \%$. Three quarters of the cases come from rural areas. Children represent few cases of HPS in the United States but account for a larger proportion of cases in South America [23].

After the 1993 Four Corners outbreak, a serosurvey was done to identify milder, unreported cases. Outpatients presenting with a syndrome of fevers and myalgias resembling the HPS prodrome were surveyed; no patient had IgM antibody reactive with SNV [24]. Recently however, five cases of acute SNV infection documented by serology were identified, with a clinical illness less severe than HPS
[25•]. Several mild and even asymptomatic cases of HPS have also been reported in South America [23].

Risk factors for HPS identified from a case-control study during the 1993 outbreak included peridomestic cleaning or agricultural activities. More rodents were trapped around the homes of case patients than the homes of controls [20].

\section{Clinical Manifestations}

The clinical manifestations of HPS can be divided into prodromal, cardiopulmonary, and convalescent phases (Fig. 2 ). In the prodromal stage, which usually lasts 3 to 6 days, the most frequent symptoms are fever, chills, and myalgias. Abdominal pain, nausea, vomiting, headache, and dizziness may occur. Respiratory symptoms are often absent. Sore throat, coryza, and meningismus are uncommon, and their presence may be useful in differentiating HPS from other viral infections. Physical examination, laboratory data, and chest radiographs may be normal in this stage.

The cardiopulmonary stage, heralded by cough and dyspnea, can progress rapidly; pulmonary edema and respiratory failure may develop within hours of presentation. Physical examination is notable for tachypnea, tachycardia, and hypotension. Chest radiographs usually show interstitial edema and basilar or central air space disease. These findings contrast with those for acute respiratory distress syndrome (ARDS), in which interstitial edema is rare and air space disease is usually peripherally distributed early in the course. Hemodynamic measures are characterized by low initial pulmonary artery occlusion pressure, high systemic vascular resistance, and depressed cardiac output, consistent with a noncardiac cause of pulmonary edema [26]. Pleural effusions may occur in up to $78 \%$ of patients [27]. The pleural fluid is initially transudative but may become exudative during recovery because fluid shifts as cardiac function normalizes. Severe cardiopulmonary dysfunction appears to predict a poor prognosis. In fatal cases, the cardiac index progressively decreases, with severe oxygen debt and elevated lactate levels. Survivors can be extubated as early as 24 to 48 hours, but extubation generally takes place between 4 and 6 days. 


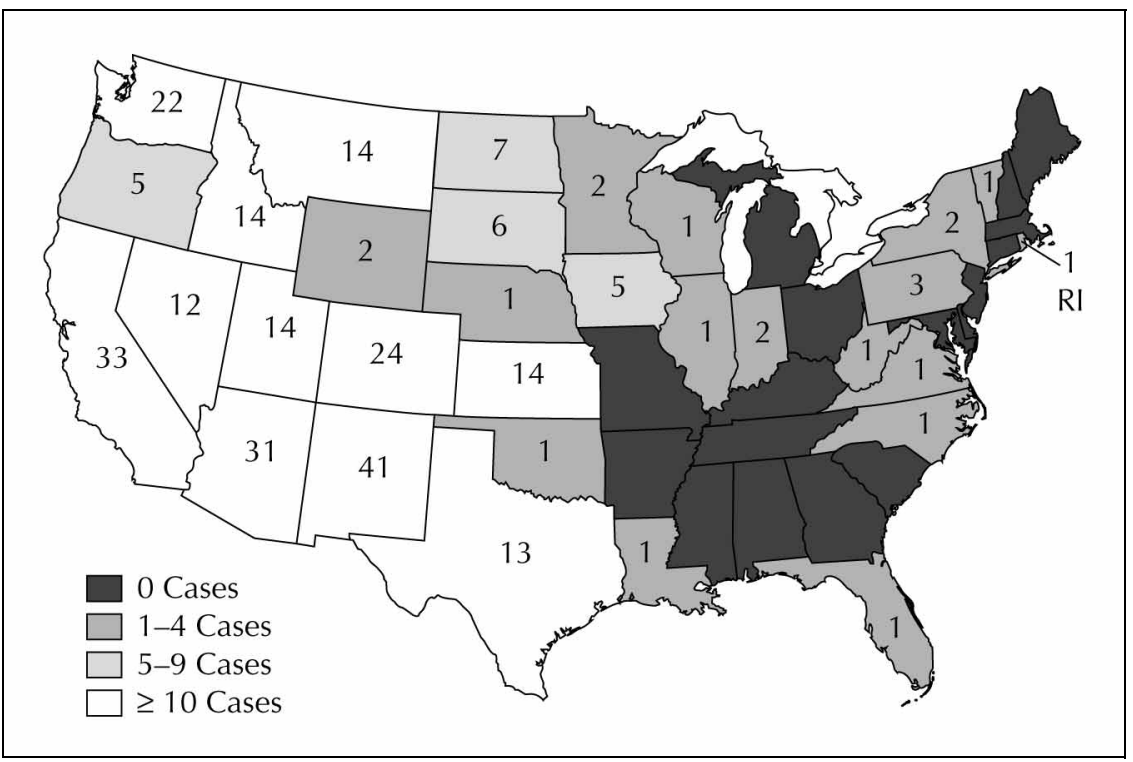

Figure 1. Hantavirus pulmonary syndrome cases by state of residence from May 1993 through December 2000. (Adapted from Centers for Disease Control and Prevention: http://www.cdc.gov/ncidod/diseases/hanta/ hps/noframes/epislides/episl7.htm.)

The classic laboratory findings in HPS are hemoconcentration and thrombocytopenia. Other laboratory findings are leukocytosis, with a left shift and atypical lymphocytosis; elevated coagulation indices (disseminated intravascular coagulation is uncommon); and elevated aminotransferase levels. Serum creatinine levels can be elevated $(<2.5 \mathrm{mg} / \mathrm{dL})$ in severe cases, but frank renal failure is uncommon. Metabolic acidosis with lactic acidemia can be seen in severe cases.

Moolenaar et al. [28] compared the clinical and laboratory characteristics of patients with HPS with those of patients with bacteremic pneumococcal pneumonia, influenza, and unexplained ARDS. The presence on hospital admission of dizziness, nausea or vomiting, and absence of cough, in association with thrombocytopenia, hemoconcentration, and acidosis, identified all patients with HPS and excluded HPS in at least $80 \%$ of patients with unexplained ARDS.

The convalescent phase of HPS is marked by diuresis, with improvement in oxygenation and decrease in hematocrit. Complete recovery can be rapid, even in patients on maximum ventilatory and inotropic support. Cognitive impairments, especially involving memory, have been described in two HPS survivors 1 year after recovery [29]. It is unclear, however, whether this is a consequence of anoxic brain damage or viral-induced brain injury.

Terajima et al. [30] used a quantitative reverse transcription polymerase chain reaction assay for SNV to analyze samples from 26 patients with HPS. Twenty of the initial 26 samples were positive for viral RNA. The mean virus copy number in positive cases was $10^{6.1 \pm 1.4} / \mathrm{mL}$. Fatal cases had one $\log _{10}$ higher mean RNA copy number compared with survivors. The viral RNA copy number correlated with the peak hematocrit value and lowest platelet count. Viremia decreased promptly after resolution of fever in eight survivors studied.
Cases of HPS caused by hantaviruses other than SNV show some clinical variations. In Black Creek Canal, Bayou, and Andes viruses, renal insufficiency and ele-

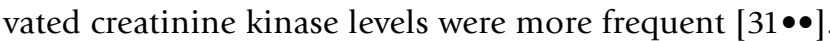
In Andes virus infection, bleeding manifestations and petechiae were also reported [23].

Cases of acute, seropositive SNV infection that do not fit diagnostic criteria for HPS have been reported [25•]. These patients presented with classic prodromal symptoms but did not progress to severe pulmonary involvement. Several of the laboratory findings associated with HPS, including thrombocytopenia, atypical lymphocytosis, and elevated aminotransferase levels, were seen. Possible explanations for the milder clinical illness include a weaker immune response to the virus, altered receptors, and virologic factors.

The differential diagnosis of HPS depends on the clinical stage of infection. The prodromal phase is indistinguishable from many other viral infections. Once infection progresses to the cardiopulmonary stage, the differential diagnosis includes leptospirosis, legionellosis, chlamydial infection, mycoplasma infection, meningococcemia, influenza, Q fever, pneumonic plague, tularemia, and disseminated fungal infections.

\section{Diagnosis}

The diagnosis of HPS is confirmed in a clinically compatible illness with serology or detection of hantaviral antigen in tissue by immunohistochemical or polymerase chain reaction. Hantavirus can be cultured, but this is time consuming and requires at least category III containment facilities. Serology detects IgM and IgG antibodies by enzyme-linked immunosorbent assay, using recombinant nucleocapsid proteins of different hantaviruses. Serum samples from acute and convalescent phases in patients 


\begin{tabular}{l}
\hline Table 3. Demographic characteristics of patients with hantavirus pulmonary syndrome \\
\begin{tabular}{|lcc|}
\hline Characteristic & Total (through I 2/7/00) & First I 00 cases (through I 2/I 5/94) \\
\hline Cases, $n$ & 277 & 100 \\
Mean age, $y$ & 38 & 39 \\
Case-fatality, $n(\%)$ & $106(38)$ & $52(52)$ \\
Male patients, $n(\%)$ & $165(60)$ & $54(54)$ \\
Race, $n(\%)$ & $2 / 4(77)$ & $63(63)$ \\
White & $55(20)$ & $35(35)$ \\
Native American & $5(2)$ & $2(2)$ \\
Black & $3(1)$ & 0 \\
Asian & $3 I(I I)$ & $9(9)$ \\
Hispanic ethnicity, $n(\%)$ & & \\
\hline
\end{tabular}
\end{tabular}

with HPS shows a fourfold increase in IgG antibody titer or the presence of IgM (acute phase) [32]. By the time clinical symptoms develop, all patients with acute SNV infection have detectable IgM antibody to the viral nucleocapsid protein. Most patients have IgG antibody directed against the nucleocapsid or G1 protein. Antibody directed against G1 membrane surface glycoprotein is highly specific to SNV infection as opposed to other hantaviral infections [33]. Among SNV-specific IgG antibodies reported in one study [34], the most prevalent were IgG3 (97\%), IgG1 $(70 \%)$, IgG2 (30\%), and IgG4 (3\%). It is postulated that the cytokines produced in SNV infection may preferentially stimulate production of IgG3 subclass antibody. In this study, antibody titers were similar in patients who died and those who survived. IgG antibody persists for years. Reinfection with homologous hantaviruses is not known to occur in humans.

The major difficulty with serologic diagnosis is the delay in obtaining results. A rapid antibody test for SNV in the form of a strip immunoblot assay has been developed; this test takes about 5 hours to complete. The assay incorporates antigens derived from synthetic peptide and recombinantexpressed forms of SNV glycoprotein and nucleocapsid protein, as well as recombinant-expressed Seoul virus nucleocapsid protein. In studies, this assay identified all patients with acute SNV infection from the earliest clinical samples with no false-positive results in controls [33].

\section{Pathology and Pathogenesis}

Gross pathologic examination of the lungs from patients with HPS shows edema and large serous pleural effusions. Microscopic examination reveals intra-alveolar edema and interstitial lymphocytic infiltrates. Minimal hyaline membrane formation and rare neutrophils help distinguish HPS from ARDS. A viral cytopathic effect is notably absent [17].

Immunohistochemical analysis has demonstrated viral antigen distributed in the capillary endothelium of multiple organs, most markedly in the pulmonary vasculature [35].
The pulmonary endothelial cells and pneumocytes show no evidence of direct viral injury, which may account for the rapid recovery observed in survivors.

The increased pulmonary capillary permeability with structurally intact cells and interstitial lymphocytic infiltrates suggests a pathogenic role for the immune system. Activated T cells, primarily $\mathrm{CD}^{+}$cells, are prominent in lung lesions and blood. It is postulated that $\mathrm{T}$ cells recognize and act on the heavily infected pulmonary cells, resulting in the secretion of inflammatory mediators such as interferon- $\gamma$ and tumor necrosis factor. These lymphokines may mediate the reversible increase in vascular permeability resulting in pulmonary edema [31••]. Mori et al. [36] demonstrated specific localization of cytokine-producing cells in the lungs of patients with HPS, paralleling the distribution of hantaviral antigens. Testing detected cells that produced both monocyte-derived cytokines (including interleukin- $1 \alpha$, interleukin-1 $\beta$, interleukin-6, and tumor necrosis factor- $\alpha$ ) and lymphocyte-derived cytokines (including interferon- $\gamma$, interleukin-2, interleukin-4, and tumor necrosis factor- $\beta$ ). Cytokine production by both activated T lymphocytes and monocytes probably contributes to the pathogenesis of the capillary leak syndrome in HPS and the renal failure observed with HFRS.

The cellular receptor for pathogenic hantaviruses has recently been identified as the $\beta$ - 3 integrins [37•]. Integrins help regulate vascular permeability and platelet function. Specific integrins expressed on platelets and endothelial cells permit entry of HPS-associated hantaviruses. The hantavirus-integrin interaction, by altering normal endothelial cell barrier functions, may be responsible for the abnormal vascular permeability associated with HPS. In tissue culture, the entry of HPS-associated hantavirus into cells was partially blocked by antibodies to the integrins, suggesting a possible future therapeutic intervention [37•].

Variation in receptor molecules is one explanation for the spectrum of illness observed with HPS [25•]. Others include specific viral differences, inoculum differences, and host factors, such as HLA haplotype [38]. Immunologic responses mediating viral clearance and recovery from HPS may also be important. In one study of patients with HPS 


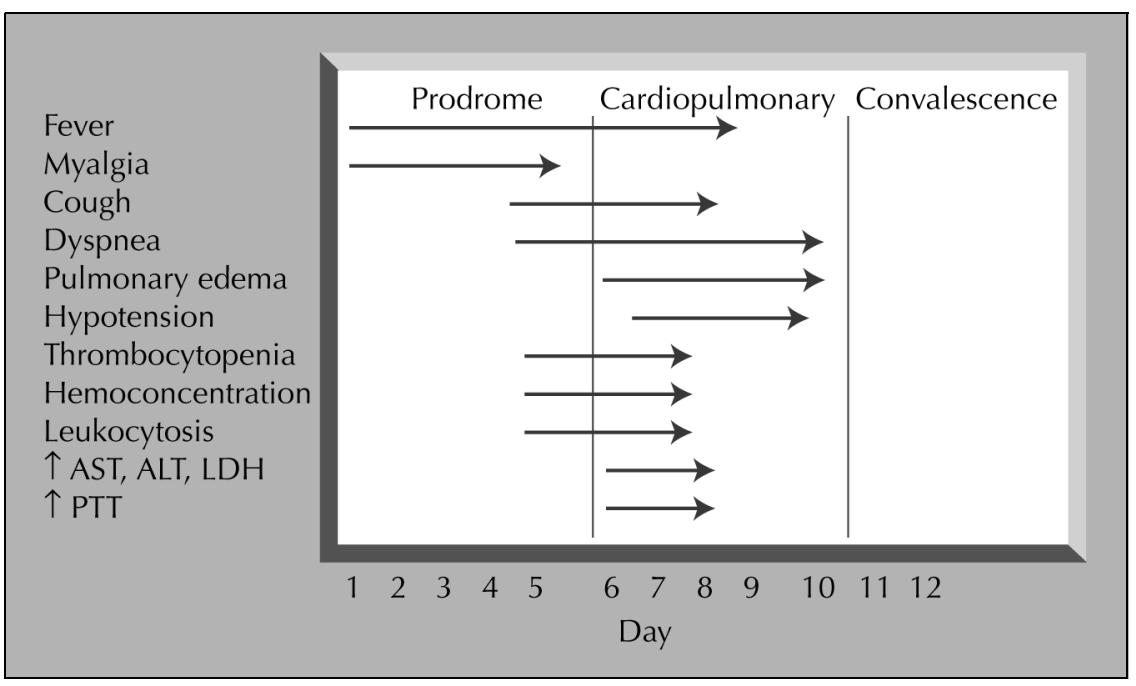

Figure 2. Clinical and laboratory features of hantavirus pulmonary syndrome. ALT—alanine aminotransferase; AST—aspartate transaminase; $\mathrm{LDH}$-lactate dehydrogenase; PTT_partial thromboplastin time.

of varying clinical severity, neutralizing antibody titers at hospital admission were lower in patients with severe disease (requiring intubation) than in patients with mild disease, suggesting a role for immunotherapy [39]. In animal models, passive administration of immunoglobulin after exposure protected against infection, but no human trials have been performed [15].

\section{Treatment}

The prodrome of HPS is indistinguishable from other viral infections. Serologic testing is usually not performed until respiratory symptoms develop, at which point progression to death can be rapid. Treatment remains primarily supportive, including attention to proper fluid administration and cardiopulmonary support. Antimicrobial therapy is often initiated for other potentially treatable diseases in the differential diagnosis.

An open-label trial of ribavirin for suspected HPS was conducted between June 1993 and September 1, 1994. Ribavirin was selected on the basis of a prospective, double-blinded, placebo-controlled trial of ribavirin for HFRS in China [40]. This study suggested that mortality was lower if the drug was administered within 4 days of diagnosis [40]. The investigators also reported that the drug had an acceptable safety profile, and they showed in vitro susceptibility to ribavirin. The study enrolled 140 patients; hantavirus infection was confirmed in 30 patients, and the mortality rate was $47 \%$. The investigators could not assess the efficacy of intravenous ribavirin, but comparison of the 30 study patients who had HPS with 34 untreated patients with HPS who were not enrolled in the study showed no difference in survival between the groups. Anemia developed in $71 \%$ of patients, $19 \%$ of whom had transfusion. In 1995, a double-blind, placebo-controlled trial of ribavirin sponsored by the National Institutes of Health, was initiated to evaluate efficacy in HPS. Patients with suspected HPS in the prodromal as well as cardiopulmonary stages are eligible $[15,40]$.
The observation that severe cardiopulmonary failure in HPS is reversible led to treatment with extracorporeal membrane oxygenation (ECMO) [41•]. The characteristic rapid recovery in the convalescent stage would only require a short duration of ECMO, which has its own related complications. Inclusion criteria for ECMO therapy were risk factors that have been shown to be associated with a $100 \%$ predicted mortality rate in a series of HPS-related deaths, including a cardiac index less than $2.5 \mathrm{~L} / \mathrm{min} / \mathrm{mm}$, lactate level greater than $4 \mathrm{mmol} / \mathrm{L}$, ventricular fibrillation or tachycardia, pulseless electrical activity, or refractory shock. Patients with irreversible neurologic or multiorgan failure or those on prolonged ventilation were generally excluded. Between 1994 and 1995, ECMO successfully provided support in two patients with HPS. A third eligible patient developed ventricular fibrillation before transfer to the ECMO unit and died. The hemodynamic improvement in the two survivors was dramatic in the first 12 hours of ECMO. Both patients were weaned off the ventilator within 4 to 5 days. Early transfer of patients with HPS to a center offering ECMO should be considered.

A 16-year-old boy with HPS was successfully treated with inhaled nitric oxide [42]. Oxygenation in this patient rapidly improved, and the boy was extubated 43 hours after initiation of therapy. Nitric oxide is a potent vascular relaxant. Local administration delivers it directly to the pulmonary vascular epithelium, leading to reduced pulmonary artery pressure; this may be the mechanism that improves pulmonary edema. Although it is difficult to reach any conclusions about efficacy of nitric oxide on the basis of a single case report, this may be a modality for future study.

\section{Prevention}

Limiting exposure to rodents and excreta is the most effective way to decrease the risk of HPS. Elimination of food sources and nesting sites for rodents is crucial. Rodenticides are useful, but fleas should be eliminated first in plague-endemic regions. Areas where rodent infestation is 
suspected should be well ventilated before humans enter. Brooms or vacuums should be avoided to prevent aerosolization of potentially infected rodent excreta. The Centers for Disease Control and Prevention has published detailed recommendations for the prevention of HPS [43].

Vaccines for hantavirus infections have been developed against strains associated with the HFRS form of disease. An inactivated Hantaan virus vaccine is licensed for use in Korea. In one study, $75 \%$ of patients receiving this vaccine produced neutralizing antibody after the first booster dose; however, the humoral response was short-lived [44]. Hantaviral G1, G2, and nucleocapsid proteins are known to be immunogenic. This finding has prompted interest in developing recombinant vaccines using baculovirus and vaccinia-expressed glycoproteins. In rodents, these vaccines could elicit neutralizing antibody responses and protect against Hantaan virus infection [45-47].

Additional studies using recombinant DNA vaccines in animal models have shown similar success. In rodent studies, use of either Seoul virus M or S genome segments in DNA vaccines elicited Seoul-specific neutralizing antibody responses; however, only $M$ segment vaccination protected rodents from infection [48]. DNA vaccines against hantaviruses that cause HPS are being developed [7].

\section{Conclusions}

The discovery of HPS in 1993 was followed by a rapid accumulation of information. Within weeks of recognition of this syndrome, the etiologic agent and its reservoir in nature were identified, and a trial of a potential therapeutic agent was initiated. New viruses continue to be discovered in both humans and rodents throughout the Americas, and milder forms of disease are now recognized. With the discovery of new viruses, questions continue to arise about the spectrum of clinical illness, routes of transmission, the role of the immune system in pathogenesis, and future treatment.

\section{References and Recommended Reading}

Papers of particular interest, published recently, have been highlighted as:

- Of importance

-• Of major importance

1. Elliot LH, Ksiazek TG, Rollin PE, et al.: Isolation of the causative agent of hantavirus pulmonary syndrome. Am J Trop Med Hyg 1994, 51:1020-1028.

2. Duchin JS, Koster FT, Peters CJ, et al.: Hantavirus pulmonary syndrome: a clinical description of 17 patients with a newly recognized disease. $N$ Engl J Med 1994, 330:949-955.

3. Khan AS, Khabbaz RF, Armstrong LR, et al:: Hantavirus pulmonary syndrome: the first 100 US cases. J Infect Dis 1996, 173:1297-1303.

4. Rollin PE, Ksiazek TG, Elliot TG, et al.: Isolation of Black Creek Canal virus, a new hantavirus from Sigmodon hispidus in Florida. J Med Virol 1995, 46:35-39.

5. Khan AS, Spiropoulou CS, Morzunov S, et al: : A fatal illness associated with a new hantavirus in Louisiana. J Med Virol 1995, 46:281-286.
6. Gavrilovskaya I, LaMonica R, Fay M-E, et al.: New York 1 and Sin Nombre viruses are serotypically distinct viruses associated with hantavirus pulmonary syndrome. J Clin Microbiol 1999, 37:122-126.

7. Schmaljohn C, Hjelle B: Hantaviruses: a global disease problem. Emerg Infect Dis 1997, 3:95-104.

8. Henderson WW, Monroe MC, St Jeor SC, et al.: Naturally occurring Sin Nombre virus genetic reassortments. Virology 1995, 214:602-610.

9. Li D, Schmaljohn AL, Anderson $\mathrm{K}$, et al.: Complete nucleotide sequences of the $M$ and $S$ segments of two hantavirus isolates from California: evidence for reassortment in nature among viruses related to hantavirus pulmonary syndrome. Virology 1995, 206:973-983.

10. Rodriguez LL, Owens JH, Peters CJ, et al.: Genetic reassortment among viruses causing hantavirus pulmonary syndrome. Virology 1998, 242:99-106.

11. Hart CA, Bennett M: Hantavirus infections: epidemiology and pathogenesis. Microbes Infect 1999, 1:1229-1237.

This review focuses on the virology, ecology, and transmission of hantaviruses.

12.• McCaughey C, Hart CA: Hantaviruses. J Med Microbiol 2000, 49:587-599.

This is an excellent comprehensive review of hantaviral infections, including a discussion of evidence of hantaviral infection in the British Isles.

13. Mills JN, Ksiazek TG, Peters CJ, et al.: Long-term studies of hantavirus reservoir populations in the Southwestern United States: a synthesis. Emerg Infect Dis 1999, 5:135-142.

14. Meyer BJ, Schmaljohn CS: Persistent hantavirus infections: characteristics and mechanisms. Trends Microbiol 2000, 8:61-67.

15. Mertz GJ, Hjelle BL, Bryan RT: Hantavirus infection. Adv Intern Med 1997, 42:369-421.

16. Schussler JM, Jordan $\mathrm{H}$, Stokoe $\mathrm{C}$, et al.: Hantavirus pulmonary syndrome. South Med J 1999, 92:233-235.

17. Butler JC, Peters CJ: Hantaviruses and hantavirus pulmonary syndrome. Clin Infect Dis 1994, 19:387-395.

18. Howard MJ, Doyle TJ, Koster FT, et al.: Hantavirus pulmonary syndrome in pregnancy. Clin Infect Dis 1999, 29:1538-1544.

This detailed review of five cases of HPS in pregnancy reported in the United States includes a literature review of hantaviral infections during pregnancy.

19. Vitek CR, Breiman RF, Ksiazek TG, et al.: Evidence against person-to-person transmission of hantavirus to health care workers. Clin Infect Dis 1996, 22:824-826.

20. Zeitz PS, Butler JC, Cheek JE: A case-control study of hantavirus pulmonary syndrome during an outbreak in the southwestern United States. J Infect Dis 1995, 171:864-870.

21. Padula PJ, Edelstein A, Miguel SDL, et al:: Hantavirus pulmonary syndrome outbreak in Argentina: molecular evidence for person-to person transmission of Andes virus. Virology 1998, 241:323-330.

This interesting study investigates an outbreak of HPS in Argentina. The authors conclude that person-to-person transmission has been demonstrated using comparative analysis between different viral sequences from human cases.

22. Centers for Disease Control and Prevention: All about hantaviruses. Accessible at http://www.cdc.gov/ncidod/ diseases/hanta/hps/noframes/caseinfo.htm. Accessed December 2000.

23. Toro J, Vega JD, Khan AS, et al:: An outbreak of hantavirus pulmonary syndrome in Chile, 1997. Emerg Infect Dis 1998, 4:687-693.

24. Simonsen L, Dalton MJ, Breiman RF, et al.: Evaluation of the magnitude of the 1993 hantavirus outbreak in the southwestern United States. J Infect Dis 1995, 172:729-733.

25. Kitsutani PT, Denton RW, Fritz CL, et al.: Acute Sin Nombre hantavirus infection without pulmonary syndrome, United States. Emerg Infect Dis 2000, 3:701-705.

This is an important case series of five patients identified in the United States with acute SNV infection that did not fit the HPS case definition. These cases established the presence of milder clinical illness. 
26. Hallin GW, Simpson SQ, Crowell RE, et al.: Cardiopulmonary manifestations of hantavirus pulmonary syndrome. Crit Care Med 1996, 24:252-258.

27. Bustamante EA, Levy H, Simpson SQ: Pleural fluid characteristics in hantavirus pulmonary syndrome. Chest 1997, 112:1133-1136.

28. Moolenaar RL, Dalton CD, Lipman HB, et al.: Clinical features that differentiate hantavirus pulmonary syndrome from three other acute respiratory illnesses. Clin Infect Dis 1996, 21:643-649.

29. Hopkins RO, Larson-Lohr V, Weaver LK, et al.: Neuropsychological impairments following hantavirus pulmonary syndrome. J Int Neuropsychol Soc 1998, 4:190-196.

30. Terajima $M$, Hendershot JD, Kariwa $H$, et al.: High levels of viremia in patients with hantavirus pulmonary syndrome. J Infect Dis 1999, 180:2030-2034.

$31 . \bullet$ Peters CJ, Simpson GL, Levy H: Spectrum of hantavirus infection: hemorrhagic fever with renal syndrome and hantavirus pulmonary syndrome. Ann Rev Med 1999, 50:531-545.

This is an excellent review of the clinical spectrum of disease and pathogenesis of hantaviral infections.

32. Jenison S, Yamada T, Morris C, et al.: Characterization of human antibody responses to Four Corners hantavirus infection among patients with hantavirus pulmonary syndrome. J Virol 1994, 68:3000-3006.

33. Hjelle B, Jenison S, Torrez-Martinez N, et al:: Rapid and specific detection of Sin Nombre virus antibodies in patients with hantavirus pulmonary syndrome by a strip immunoblot assay suitable for field diagnosis. J Clin Microbiol 1997, 35:600-608.

34. Bostik P, Winter J, Ksiazek TG, et al:: Sin Nombre virus (SNV) Ig isotype antibody response during acute and convalescent phases of hantavirus pulmonary syndrome. Emerg Infect Dis 2000, 6:184-187.

35. Butler JC, Zaki SR, Khabbaz RF, et al.: Hantavirus pulmonary syndrome. Infect Dis Clin Pract 1995, 4:189-192.

36. Mori M, Rothman AL, Kurane I, et al.: High levels of cytokineproducing cells in the lung tissues of patients with fatal hantavirus pulmonary syndrome. J Infect Dis 1999, 179:295-302.

37. Gavrilovskaya IN, Shepley M, Shaw R, et al.: B3 integrins mediate the cellular entry of hantaviruses that cause respiratory failure. Proc Natl Acad Sci U S A 1998, 95:7074-7079.

This study identifies the $\beta-3$ integrins as the cellular receptors for hantaviruses and investigates the roles of different integrins in facilitating the entry of pathogenic versus nonpathogenic strains of hantavirus.
38. Ennis FA, Cruz J, Spiropoulou CF, et al.: Hantavirus pulmonary syndrome: CD4 and CD8 cytotoxic T lymphocytes to epitopes on Sin Nombre Virus nucleocapsid protein isolated during acute illness. Virology 1997, 238:380-390.

39. Bharadwaj M, Nofchissey R, Goade D, et al.: Humoral immune responses in the hantavirus pulmonary syndrome. J Infect Dis 2000, 182:43-48.

40. Chapman LE, Mertz GJ, Peters CJ, et al.: Intravenous ribavirin for hantavirus pulmonary syndrome: safety and tolerance during 1 year of open-label experience. Antiviral Ther 1999, 4:211-219.

41. Crowley MR, Katz RW, Kessler R, et al.: Successful treatment of adults with severe hantavirus pulmonary syndrome with extracorporeal membrane oxygenation. Crit Care Med 1998, 26:409-414.

This is a review of the use of ECMO in three patients with HPS. It discusses the rationale for use, inclusion criteria, clinical course, and outcome.

42. Rosenberg RB, Waagner DC, Romano MJ, et al:: Hantavirus pulmonary syndrome treated with inhaled nitric oxide. Pediatr Infect Dis J 1998, 17:749-752.

43. Hantavirus infection-southwestern United States. Interim recommendations for risk reduction. MMWR Morb Mortal Wkly Rep 1993, 42(RR-11):1-13.

44. Cho H-W, Howard CR: Antibody responses in humans to an inactivated hantavirus vaccine. Vaccine 1999, 17:2569-2575.

45. Chu YK, Jennings GB, Schmaljohn CS: A Vaccinia virusvectored Hantaan virus vaccine protects hamsters from challenge with Hantaan and Seoul viruses but not Pumaala virus. J Virol 1995, 69:6417-6423.

46. Schmaljohn CS, Chu YK, Schmaljohn AL, et al.: Antigenic subunits of Hantaan virus expressed by baculovirus and vaccinia virus recombinants. J Virol 1990, 64:3162-3170.

47. Xu X, Ruo SL, McCormick JB, et al.: Immunity to Hantavirus challenge in Meriones unguicalatus induced by vacciniavectored viral proteins. Am J Trop Med Hyg 1992, 47:397-404.

48. Hooper JW, Kamrud KI, Elgh F, et al.: DNA vaccination with hantavirus $M$ segment elicits neutralizing antibodies and protects against Seoul virus infection. Virology 1999, 255:269-278.

49. Update: hantavirus pulmonary syndrome - United States, 1993. MMWR Morbid Mortal Wkly Rep 1993, 42:816-820. 